\title{
Modeling Morphogenesis in Multicellular Structures with Cell Complexes and L-systems
}

\author{
Przemyslaw Prusinkiewicz and Brendan Lane
}

\begin{abstract}
We consider computational modeling of biological systems that consist of discrete components arranged into linear structures. As time advances, these components may process information, communicate and divide. We show that: (1) the topological notion of cell complexes provides a useful framework for simulating information processing and flow between components; (2) an index-free notation exploiting topological adjacencies in the structure is needed to conveniently model structures in which the number of components changes (for example, due to cell division); and (3) Lindenmayer systems operating on cell complexes combine the above elements in the case of linear structures. These observations provide guidance for constructing L-systems and explain their modeling power. L-systems operating on cell complexes are illustrated by revisiting models of heterocyst formation in Anabaena and by presenting a simple model of leaf development focused on the morphogenetic role of the leaf margin.
\end{abstract}

\section{Introduction}

There is a feedback between mathematics and studies of nature. On one hand, mathematical concepts - even though they may eventually be formalized in an axiomatic way - are often inspired and motivated by studies of nature. On the other hand, they facilitate these studies by providing proper mathematical tools (Fig. 1).

In this context, we consider computational methods needed to model the development of multicellular structures, in particular plants. We show that these methods are not merely a new application of partial differential equations, traditionally used to model spatio-temporal phenomena in mathematical physics. Instead, developmental modeling of multicellular structures requires an integration of tools rooted in different branches of mathematics and computer science. This combination includes L-systems [1], ordinary differential equations, and the topological notion of cell complexes [2]. 
Fig. 1: A conceptual model of relations between natural science and mathematics.

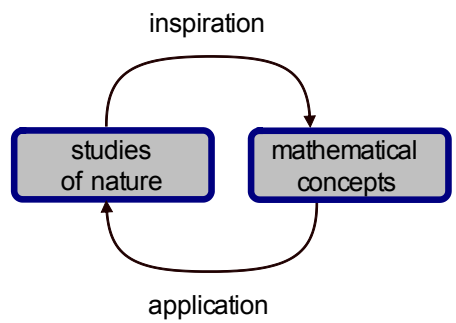

The structures we consider are the spatial arrangements of discrete components that process information and communicate. These structures are dynamic, which means that not only the state of the components, but also their number can change over time. The development is symplastic: the neighborhood relations can only be changed as a result of the addition or removal of components (in contrast to animal cells, plant cells do not move with respect to each other). We limit our examples to linear structures consisting of sequences of cells, although similar problems occur in the modeling of branching plant structures at the larger scale of architectural modules: branch and root segments, buds, leaves, flowers and fruits. The insights we obtain also extend to models of two- and three-dimensional structures.

\section{Computation in Cell Complexes}

Let us consider the fundamental process of diffusion in a filament as a running example. At any point in time, the distribution of the diffusing substance can be visualized by plotting concentration $c$ as a function of position $x$ along the filament (Fig. 2a):

How can we model changes in concentration due to diffusion over time? The first impulse may be to apply the well known partial differential equation for diffusion:

$$
\frac{\partial c}{\partial t}=D \frac{\partial^{2} c}{\partial x^{2}}
$$

Unfortunately, there is a problem with this approach. To derive partial differential equation (1), one starts with a discrete description of diffusion, then passes to the limit in space and time ([3], Chap. 9). When ascribing this equation to a multicellular structure, we go back to the discrete version. Such circular thinking should be avoided [4].

One step towards a solution is to ignore (1) and directly write the set of ordinary differential equations that describe the changes in concentration in each cell without going to the spatial limit (Fig. 2b):

$$
\frac{d c_{i}}{d t}=k_{i}\left(c_{i-1}-c_{i}\right)-k_{i+1}\left(c_{i}-c_{i+1}\right), \quad i=2,3, \ldots, n-1 .
$$




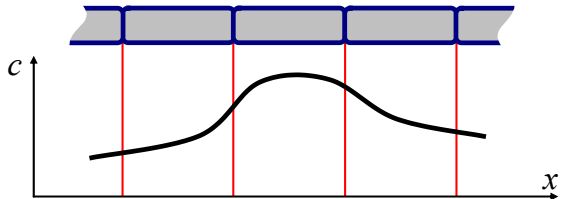

(a)

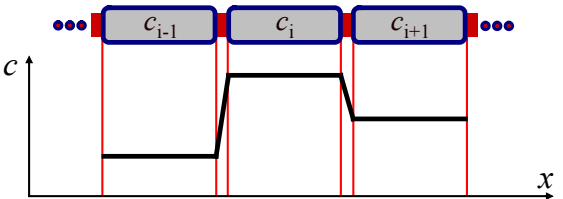

(b)

Fig. 2: Sample distribution of a diffusing substance in a spatially continuous (a) and spatially discrete (b) model of a multicellular filament.

According to this system of equations, the concentration of the diffusing substance in each of the $n-2$ cell interior to the filament changes proportionally to the difference in concentrations across cell walls (a version of Fick's law [3]). For simplicity, we do not consider here the boundary cells 1 and $n$. We assume that the concentration of the diffusing substance is approximately uniform within each cell. This is a reasonable assumption as it is the cell walls, rather than the cells themselves, that present a significant obstacle to diffusion. The system of equations (2) highlights, however, another problem, which becomes apparent when we compare the equations for adjacent cells, e.g. $i$ and $i+1$ :

$$
\begin{aligned}
\frac{d c_{i}}{d t} & =k_{i}\left(c_{i-1}-c_{i}\right)-k_{i+1}\left(c_{i}-c_{i+1}\right) \\
\frac{d c_{i+1}}{d t} & =k_{i+1}\left(c_{i}-c_{i+1}\right)-k_{i+2}\left(c_{i+1}-c_{i+2}\right)
\end{aligned}
$$

The term $k_{i+1}\left(c_{i}-c_{i+1}\right)$ is calculated twice, first to determine the amount of the diffusing substance exported from cell $i$ to cell $i+1$, and a second time to determine the amount of the substance received by cell $i+1$ from cell $i$. Superficially, performing the same calculation twice may seem merely redundant: computationally inefficient, but without any effect on the final result. However, the problem created by repeating this calculation is deeper. Suppose that the diffusion coefficients are random variables, which is well justified if the number of diffusing molecules is small. Calculating an expression with random variables twice will likely produce different results, and the amount of substance exported by cell $i$ will be different from the amount received by cell $i+1$, violating the law of mass conservation.

We can solve this problem by computing fluxes between any pair of adjacent cells only once and using the result twice, to update concentration in each cell. While implementing this solution, we need to properly recognize the topology of the modeled structure, which is a sequence of cells separated by walls. This topology offers placeholders for all variables inherent in diffusion: concentrations $c$ are associated with cells, and fluxes $J$ with walls (Fig. 3). The system of equations (4) results: 
Fig. 3: Modeling a filament as a cell complex.

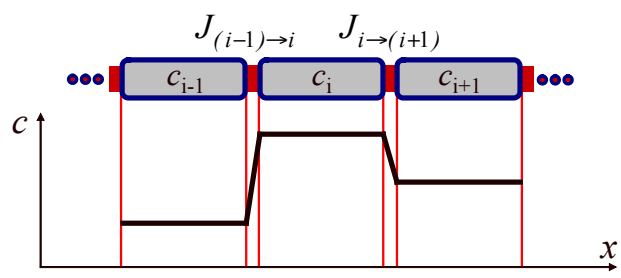

$$
\left.\begin{array}{rl}
J_{(i-1) \rightarrow i} & =K_{(i-1) \rightarrow i}\left(c_{i-1}-c_{i}\right) \\
\frac{d c_{i}}{d t} & =J_{(i-1) \rightarrow i}-J_{i \rightarrow(i+1)}
\end{array}\right\} \quad i=2,3, \ldots, n-1
$$

Although the flux through each wall is computed the same way as in (2) and (3), it is now computed only once. Consequently, mass is conserved even in the presence of random fluctuations of flux. Furthermore, if the system of equations (4) is evaluated numerically, for example using the forward Euler method with time step $\Delta t$

$$
\left.\begin{array}{rl}
J_{(i-1) \rightarrow i}^{t+1} & =K_{(i-1) \rightarrow i}^{t}\left(c_{i-1}^{t}-c_{i}^{t}\right) \\
c^{t+1} i & =c_{i}^{t}+\left(J_{(i-1) \rightarrow i}^{t}-J_{i \rightarrow(i+1)}^{t}\right) \Delta t
\end{array}\right\} \begin{aligned}
& i=2,3, \ldots, n-1 \\
& t=0,1,2, \ldots
\end{aligned}
$$

mass will be conserved exactly in spite of the errors in estimating fluxes over finite time intervals that are inherent in numerical methods.

A sequence of cells separated by walls is an example of a one-dimensional cell complex [2]. Formally, such a complex is an interwoven sequence of objects of two types: line segments (cells) and points at which these segments meet (walls). Thinking in terms of cell complexes facilitates proper definition of discrete models, especially in higher dimensions. For example, in three-dimensional tissues built from polyhedral cells we distinguish three-dimensional cells, two-dimensional polygonal faces that are shared by pairs of cells, one-dimensional edges that bind these faces, and zero-dimensional vertices in which the edges meet. Each of these objects may provide a placeholder for different variables, organizing simulations of multicellular organisms in a systematic manner [5].

\section{L-systems}

Do cell complexes provide a good framework for describing processes such as diffusion or genetic regulation in multicellular systems? They are certainly a step in the right direction, but many problems remain. The key issue is how to identify the components of a cell complex, the variables related to each component, and the equations that relate these variables. One method is to use indices and specify neighborhood relations between the components with index arithmetic. For instance, if $n$ components are arranged into a sequence indexed from 1 to $n$, the neighbors of component $i \in\{2, \ldots, n-1\}$ will have indices $i-1$ and $i+1$. Equations (4) and (5) 


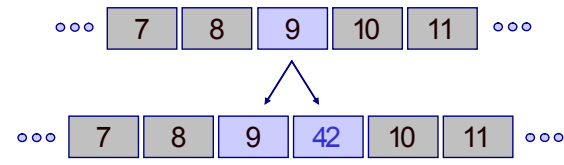

(a)

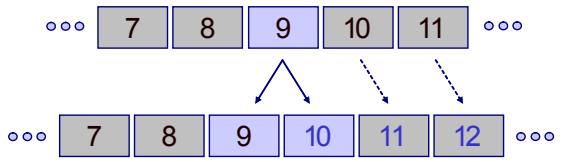

(b)

Fig. 4: Inadequacies of cell identification with indices in a growing filament. (a) Assignment of an arbitrary number to a newly created cell breaks index arithmetic. (b) Renumbering dissociates indices from the identity of cells.

are examples of this notation. It is so standard in mathematical practice that we tend to use it without much thought. Unfortunately, it does not work well for developing systems.

To see the problem, let us consider a filament with consecutively numbered cells [6]. A cell, say number 9, divides and becomes two cells. What indices should they have? One possibility would be to pass number 9 to one of the child cells and assign some arbitrary number that has not yet been used, say 42 , to the other cell (Fig. 4a). Each cell will then have a unique identifier, but we can no longer rely on index arithmetic to find who is the neighbour of whom: it no longer suffices to add one to find the neighbour to the right, or subtract one to find the neighbour to the left.

Another possibility is to preserve index arithmetic (Fig. 4b). We can accomplish this, for example, by assigning the second new cell number 10, and renumbering all of the remaining cells to the right. The old cells 9-10-11 now become cells 10-11-12. In this case, we can perform index arithmetic on the new filament, but the identity of cells is no longer maintained. For example, cell 10 has become cell 11, and may become cell 12, 13 or higher in the future.

Analyzing these problems, we conclude that their source is not merely one or another indexing scheme, but the very attempt to use indices to identify cells in a growing organism. Paraphrasing Hermann Weyl, who said "The introduction of numbers as coordinates [...] is an act of violence" [7, p. 90], we can say "...and so is the introduction of indices."

An alternative idea is to exploit the topological structure of the filament and introduce operators that will return the informational content of the neighbours. The possibility of accessing such context in a local manner, without globally indexing all components of the modelled structure, is one of key ideas behind L-systems, the formalism for describing and simulating development introduced in 1968 by Aristid Lindenmayer [1]. Using the notation for (context-sensitive) L-systems presented in [8], we can write (5) as:

$$
\begin{gathered}
C\left(c_{L}\right)<W(J)>C\left(c_{R}\right) \rightarrow W\left(K \cdot\left(c_{L}-c_{R}\right)\right) \\
W\left(J_{L}\right)<C(c)>W\left(J_{R}\right) \rightarrow C\left(c+\left(J_{L}-J_{R}\right) \Delta t\right)
\end{gathered}
$$

L-system expressions are called rewriting rules or productions, as in the theory of algorithms and formal languages. The first production above states that wall $W$ 
is associated with a single variable, noted $J$. This variable represents flux though the wall. Its value is updated (arrow $\rightarrow$ ) considering concentrations $c_{L}$ and $c_{R}$ of the diffusing substance in cells $C$ on the left and right side of wall $W$. These cells are indicated by the operators $\langle$ and $\rangle$, respectively. The variable identifiers $c_{L}$ and $c_{R}$ are local to this production and can be arbitrary, but must be distinct. Given the wall and its context (i.e., the adjacent cells), the updated flux is calculated using the expression $K \cdot\left(c_{L}-c_{R}\right)$.

The second production works in a similar way. In order to update the concentration $c$ of the substance in cell $C$, the walls $W$ that delimit this cell on the left and right sides are considered. Each wall is characterized by a flux: $J_{L}$ and $J_{R}$, respectively. Within time $\Delta t$ associated with a single simulation step, concentration $c$ changes by $\left(J_{L}-J_{R}\right) \Delta t$. The L-system productions (6) thus express the same idea as Equation (5), but without involving indices or any other global enumeration of cells and walls. Instead, they use the operators that look for the context, or neighbourhood, of cells and walls.

Summarizing the ideas presented so far, we have introduced three notions of key importance to the modeling of multicellular structures. First, we formalized these structures as cell complexes. This notion provides a vehicle for assigning variables to proper elements of the structure, in our example concentrations to cells and fluxes to walls. Second, we used locally defined context, rather than globally defined indices (or any other global enumeration) to access information about the neighbours of any element in the complex (Figs. 4a and 4b). The third point is a little more subtle, but equally true: the arrow is an operator that relates what was before to what will come next, and thus indicates the neighbourhood in time. Thus, in contrast to Equation (5), L-system (6) needs no indices for time as well. An additional benefit of L-systems is that they naturally extend to another type of productions, which capture cell division. For example, the following production:

$$
C(c): \text { condition } \rightarrow C(c) W(0) C(c)
$$

says that, if some condition is met, cell $C$ will divide into two child cells with the same concentration $\mathrm{c}$ as the parent cell, separated by a wall.

\section{Heterocyst Differentiation in Anabaena}

To illustrate the presented concepts in a biological context, we will apply them to model morphogenesis in a growing filament. The chosen organisms, representing genus Anabaena, integrate some of the most fundamental processes linking patterning and growth. Consequently, they have been repetitively used to illustrate both the basic mechanisms of morphogenesis and diverse aspects of modeling with L-systems [9-15]. Here we focus on the integration of L-systems and cell complexes.

Anabaena is a genus of cyanobacteria, organisms that have been on Earth for over 3 billion years and are responsible for the introduction of oxygen into the atmo- 
sphere [16]. It creates multicellular filaments consisting of two basic types of cells. Vegetative cells are capable of photosynthesis and produce sugars. Heterocysts are capable of fixing nitrogen from the atmosphere, and produce nitrogenous compounds that the bacterium needs. Photosynthesis and nitrogen fixation are biologically difficult to reconcile, because the enzyme crucial to the fixation is inhibited by oxygen. Consequently, some cyanobacteria separate photosynthesis and nitrogen fixation in time: they photosynthesize during the day and fix nitrogen at night. Others, including Anabaena, separate these tasks in space [16].

On the average, consecutive heterocysts in an Anabaena filament are separated by about ten vegetative cells [17]. Heterocysts cannot divide, but vegetative cells do divide and grow, causing the filament to elongate. As the existing heterocysts are moved apart by the growing vegetative segments, new heterocysts differentiate inbetween. The process that controls this differentiation has been extensively studied. A small protein called PatS is produced by heterocysts and diffuses through the vegetative segments of the filament, inhibiting the differentiation of new heterocysts [18]. As the existing heterocysts move apart, the concentration of PatS in the vegetative segments gradually decreases, eventually falling below a threshold near the center of the segment. This decline is detected by the genetic regulatory circuit that triggers the differentiation of a new heterocyst.

The maintenance of approximately constant spacing between heterocysts in a growing filament can be captured and explained using computational models that may represent different tradeoffs between biological accuracy and simplicity. Here we present a very simple L-system illustrating the use of cell complexes.

\begin{tabular}{llll}
\hline \#define & $H$ & 0 & // Heterocyst cell type \\
\#define & $V$ & 1 & // Vegetative cell type \\
\#define & $K$ & $(\mathbf{r a n}(2.0))$ & // Diffusion coefficient \\
\#define & $V$ & 0.5 & // Turnover rate \\
\#define & $R$ & 1.1 & // Cell growth factor \\
\#define & $\Theta$ & 0.1 & // Threshold for heterocyst differentiation \\
\#define & $s_{M A X}$ & 0.8 & // Cell size at division \\
\#define & $\Delta t$ & 0.01 & // Time step \\
\hline
\end{tabular}

Axiom: $\boldsymbol{C}(H, 1,1) \boldsymbol{W}(0) \boldsymbol{C}(V, 1,1) \boldsymbol{W}(0) \boldsymbol{C}(H, 1,1)$

$p_{1}: \quad \boldsymbol{C}\left(a_{L}, c_{L}, s_{L}\right)<\boldsymbol{W}(J)>\boldsymbol{C}\left(a_{R}, c_{R}, s_{R}\right) \rightarrow \boldsymbol{W}\left(K \cdot\left(c_{L}-c_{R}\right)\right)$

$p_{2}: \boldsymbol{C}(a, c, s): a=H \rightarrow \boldsymbol{C}(H, 1,1)$

$p_{3}: \quad \boldsymbol{W}\left(J_{L}\right)<\boldsymbol{C}(a, c, s)>\boldsymbol{W}\left(J_{R}\right):$

$\left\{c \leftarrow c+\left(\left(J_{L}-J_{R}\right)-v c\right) \Delta t ; \quad s \leftarrow s R^{\Delta t} ;\right\}$

$c<\Theta \rightarrow C(H, 1,1)$

$p_{4}: \quad \boldsymbol{W}\left(J_{L}\right)<\boldsymbol{C}(a, c, s)>\boldsymbol{W}\left(J_{R}\right):$

$\left\{c \leftarrow c+\left(\left(J_{L}-J_{R}\right)-v c\right) \Delta t ; \quad s \leftarrow s R^{\Delta t} ;\right\}$

$s>s_{\text {MAX }} \rightarrow \boldsymbol{C}(V, c, s / 2) \boldsymbol{W}(0) \boldsymbol{C}(V, c, s / 2)$

$p_{5}: \quad \boldsymbol{W}\left(J_{L}\right)<\boldsymbol{C}(a, c, s)>\boldsymbol{W}\left(J_{R}\right):$

$\left\{c \leftarrow c+\left(\left(J_{L}-J_{R}\right)-v c\right) \Delta t ; \quad s \leftarrow s R^{\Delta t} ;\right\}$

$\rightarrow \boldsymbol{C}(V, c, s)$ 

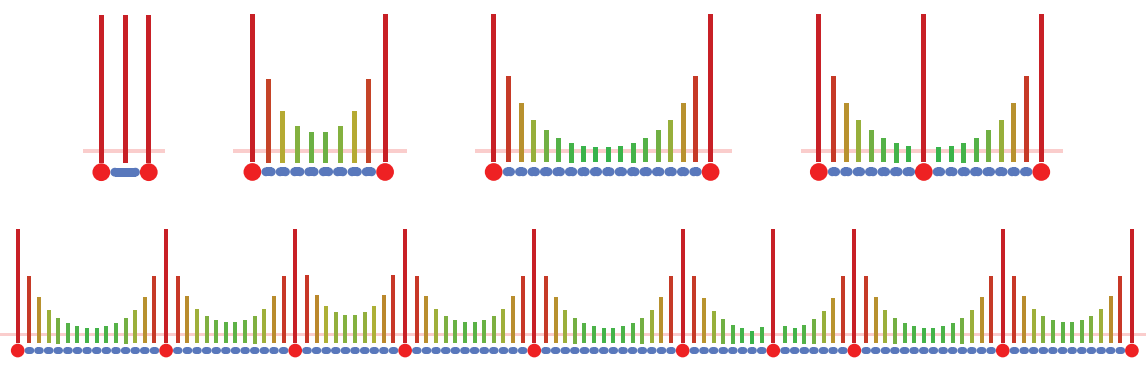

Fig. 5: Snapshots of a simulation of heterocyst differentiation in a growing Anabaena filament. Non-differentiated vegetative cells are shown in blue and heterocysts are shown in red. Vertical bars indicate concentration of a diffusing inhibitor produced by the heterocysts. The red horizontal line indicates the threshold of heterocyst differentiation.

The axiom specifies that the initial structure consists of three cells $C$ separated by walls $W$. The cells are characterized by three parameters: type $a$ ( $H$ for heterocyst, $V$ for a vegetative cell), inhibitor concentration $c$, and cell length $s$. Productions are ordered, and the first applicable production is used for each cell or wall. Production $p_{1}$ determines flux $J$ of the inhibitor across a wall, as in L-system (6). Production $p_{2}$, applicable to heterocysts, sets both the inhibitor concentration and heterocyst length to 1 . Productions $p_{3}$ to $p_{5}$ apply to vegetative cells. They describe changes in inhibitor concentration due to its diffusion and turnover, and changes in cell length due to growth. In addition, production $p_{3}$ specifies that the vegetative cell in which the inhibitor concentration falls below threshold $\Theta$ will differentiate into a heterocyst. Likewise, production $p_{4}$ states that a vegetative cell which exceeds maximum length $s_{M A X}$ will divide.

Fig. 5 shows selected steps of a simulation using this L-system. As time progresses, the vegetative cell and its descendants divide, pushing the heterocysts apart. Concentrations of the inhibitor in the vegetative cells decreases with their distance from the heterocysts, as the diffusive supply of the inhibitor diminishes. When the concentration of the inhibitor falls below a threshold, the corresponding cell differentiates into a heterocyst. The average distance between heterocysts is thus maintained in spite of the filament's growth.

\section{Leaf Development}

The previous example was focused on the arrangement of cells of different types within a growing filament. This filament was visualized by placing cells along a line, as its overall form was not important. In the next example we use a more complex geometric interpretation of an L-system operating on a cell complex. The resulting computational model plausibly explains the form of a lobed leaf. 
Fig. 6: A conceptual model of auxin flow in a growing leaf. Polarized auxin transport at the leaf margin (black arrows) leads to the emergence of auxin convergence points (blue rectangles). Canalized auxin flow from these points defines the pattern of main veins.
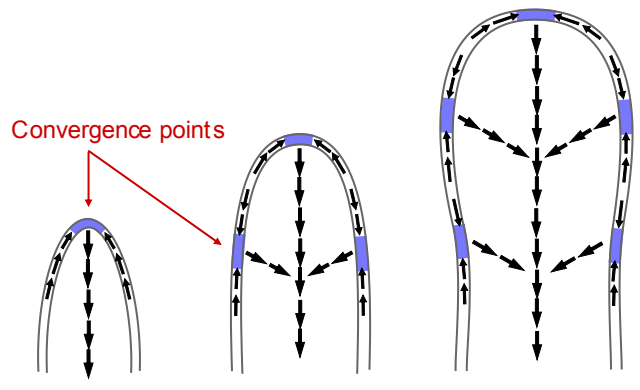

Many aspects of plant development are regulated by the plant hormone auxin [19], which is actively exported from cells by a family of proteins called PINFORMEDin short, PIN. Within this family, the PIN1 protein [20] plays the dominant role in leaf development. Confocal microscopy images of diverse plants show that PIN1 proteins at the leaf margin export auxin towards discrete locations, called convergence points $[21,22]$. From there, auxin propagates into the leaf blade, forming streams, or canals, which define the paths of future veins [23]. The first convergence point in a young leaf primordium is at the leaf tip. As the leaf grows, the distance between this convergence point and the leaf base increases, and new convergence points are gradually formed in the available space [23] (Fig. 6).

The above process can be compared to the differentiation of heterocysts in the growing filament of Anabaena. However, while the positioning of new heterocysts can be intuitively explained by the depletion of the diffusively transported inhibitor (PatS) between heterocysts moving apart, the molecular mechanism defining the spacing of convergence points on the leaf margin is not yet fully understood. The key assumption is that the concentration of PINs in the membrane of cells at the leaf margin depends on the concentration of auxin in the adjacent cells. The higher this concentration, the more PINs will be allocated to the abutting cell membrane [24, 25]. This feedback between PINs and auxin is illustrated in Fig. 7.

Here cells are represented schematically as black contours, with auxin concentrations shown as filled blue squares. The size of these squares is proportional to the concentration of auxin within the cells. Auxin fluxes are shown as black arrows between the cells: the wider the arrow, the larger the flux. PIN concentrations are visualized as red rectangles running parallel to cell edges; the wider the line, the larger the PIN concentration at the corresponding cell membrane. The feedback loop of interactions is indicated by the green arrows. The top arrow shows that PINs in the membrane of cell $i$ abutting cell $j$ pump auxin towards cell $j$. The bottom arrow shows that the concentration of auxin in cell $j$ affects the allocation of PINs in the membrane of cell $i$, and thus controls further flow of auxin into cell $j$.

To show that the postulated interactions between auxin and PINs can produce a pattern of approximately equidistant convergence points in a file of cells, we construct a simple computational model governed by three equations (for related models and their analysis see [23-27]). The first equation describes the flux $J_{i \rightarrow j}$ of auxin from cell $i$ to the adjacent cell $j$ as the sum of active and diffusive transport: 
Fig. 7: Up-the-gradient model of PIN polarization and auxin transport. Green arrows indicate the feedback of interactions: PINs (red rectangle) promote auxin efflux from cell $i$ (black arrow), while high auxin concentration in cell $j$ (blue area) polarizes PINs in cell $i$ toward $j$.

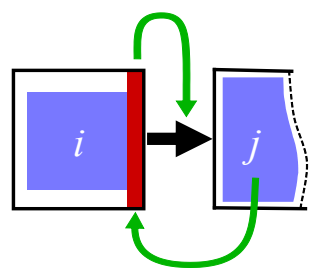

$$
J_{i \rightarrow j}=T c_{i}[P I N]_{i \rightarrow j}-T c_{j}[P I N]_{i \leftarrow j}+K\left(c_{i}-c_{j}\right) .
$$

The active transport from cell $i$ to cell $j$ is assumed to be proportional to the auxin concentration $c_{i}$ in cell $i$, multiplied by the concentration $[P I N]_{i \rightarrow j}$ of PINs in the membrane of cell $i$ abutting cell $j$. The coefficient of proportionality is $T$. An analogous term describes active transport of auxin from cell $j$ to cell $i$. The last term represents diffusive transport with the diffusion coefficient $K$, as in Equation (4, top). The second equation describes the allocation of PINs to the membrane of cell $i$ abutting cell $j$. It has the form

$$
[P I N]_{i \rightarrow j} \sim[P I N]_{i} \cdot f\left(c_{j}\right)
$$

where $[P I N]_{i}$ is the overall concentration of PINs in cell $i$, and $f$ is some increasing function of auxin concentration $c_{j}$ in cell $j$.

The third equation adds to the law of mass conservation (Equation (4), bottom) terms representing local auxin production with a constant absolute rate $\sigma$ and local turnover with relative rate $\mu$ :

$$
\frac{d c_{i}}{d t}=J_{i-1 \rightarrow i}-J_{i \rightarrow i+1}+\sigma-\mu c_{i}
$$

To model a sequence of cells and walls obeying the above equations, we express them as an L-system operating on a cell complex:

\begin{tabular}{llll}
\hline \#define & $T$ & 1.2 & // Polar transport coefficient \\
\#define & $K$ & 0.02 & // Diffusion coefficient \\
\#define & $\sigma$ & 0.1 & // Auxin production \\
\#define & $\mu$ & 0.005 & // Auxin turnover \\
\#define & $\Delta t$ & 0.05 & // Time step \\
\hline
\end{tabular}

Axiom: $\boldsymbol{C}(0,0,0) \boldsymbol{W}(0) \boldsymbol{C}(0,0,0) \cdots \boldsymbol{C}(0,0,0) \boldsymbol{W}(0) \boldsymbol{C}(0,0,0)$

$p_{1}: \quad \boldsymbol{C}\left(\overleftarrow{p_{L}}, c_{L}, \overrightarrow{p_{L}}\right)<\boldsymbol{W}(J)>\boldsymbol{C}\left(\overleftarrow{p_{R}}, c_{R}, \overrightarrow{p_{R}}\right)$

$$
\rightarrow \boldsymbol{W}\left(T\left(c_{L} \overrightarrow{p_{L}}-c_{R} \overleftarrow{p_{R}}\right)+K\left(c_{L}-c_{R}\right)\right)
$$

$p_{2}: \boldsymbol{C}\left(\overleftarrow{p_{L}}, c_{L}, \overrightarrow{p_{L}}\right) \boldsymbol{W}\left(J_{L}\right)<\boldsymbol{C}(\overleftarrow{p}, c, \vec{p})>\boldsymbol{W}\left(J_{R}\right) \boldsymbol{C}\left(\overleftarrow{p_{R}}, c_{R}, \overrightarrow{p_{R}}\right):$

$$
\rightarrow \boldsymbol{C}\left(f\left(c_{L}\right), c+\left(J_{L}-J_{R}+\sigma-\mu c\right) \Delta t, f\left(c_{R}\right)\right)
$$




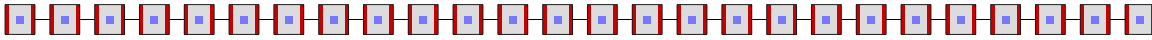

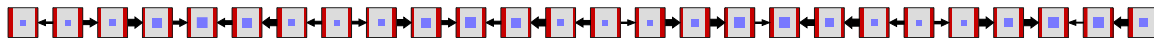

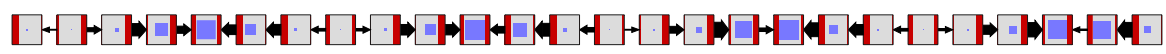

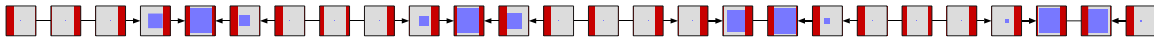

Fig. 8: The initial state and three stages of simulation of convergence point formation in a file of cells.

Fig. 9: Shape formation through boundary propagation. The outer shape results from the propagation of the inner shape in the normal directions, with a variable velocity depicted by red arrows.

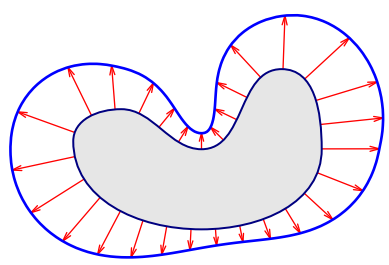

Assuming uniform distribution of auxin throughout the file of cells, the initial state of the system can be visualized as the first row of Fig. 8. As expected, a stable pattern of discrete convergence points - maxima of auxin concentration with PINs oriented towards them-emerges as the simulation progresses. Three stages of simulation in a file of constant length are shown in Fig. 8.

We will now apply the above process to model the development of leaf form. Hay et al. [21] postulated that the convergence points on the leaf margin define the positions of accelerated leaf outgrowth. A limited but simple method for modeling such outgrowth is the boundary propagation method ([28], Chap. 1). It operates by moving the boundary of a shape in the normal direction in each simulation step (Fig. 9).

We model the leaf margin as a single file of cells, initially in a shape resembling a leaf primordium. The propagation rate of each cell is proportional to the concentration of auxin. In addition, we assume that cells reaching the threshold length divide as in the case of Anabaena. Fig. 10 shows an example of the resulting progression of the shapes of the growing margin and compares the final stage of the simulation with an ivy leaf.

The molecular details of ivy leaf development are not yet known. Nevertheless, a closely related model has been constructed and supported by experiments for Arabidopsis leaves [23] and it is likely that it extends to other plants, such as ivy. In summary, both the model of heterocyst differentiation in Anabaena and the model of leaf development illustrate the principles of computational model construction using L-systems and cell complexes. In spite of their simplicity, these models provide insights into pattern formation in nature. 


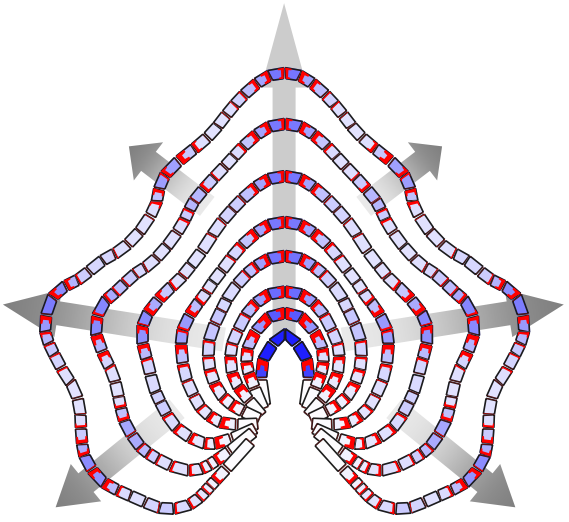

(a)

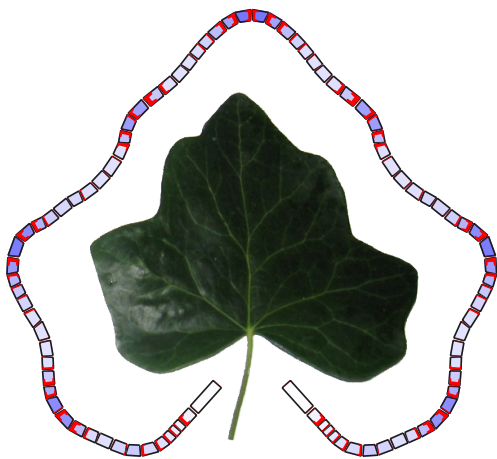

(b)

Fig. 10: A model of leaf development using the boundary propagation method. (a) Superposition of selected simulation steps. Propagation speed is controlled by auxin concentration and is the highest at the convergence points. (b) Comparison of the final shape generated by the model with an ivy leaf.

\section{Conclusions}

Mathematics of multicellular development. Modeling multicellular systems in development requires a spatially discrete formalism, which sets it apart from the continuous treatment of time and space in classical mathematical physics. Cell complexes provide a convenient abstraction for representing topological relations between components of discrete structures. Variables describing a system can be associated in a natural manner with components of different dimensions within a cell complex, allowing for convenient storage of these variables in their topologically meaningful location [29]. Respecting the principle of locality, equations relating these variables may only refer to the variables in the neighboring components of the structure. The notion of cell complexes makes it possible to access these variables using an index-free notation. This is particularly important when dealing with systems in which structure changes dynamically, for example as a result of cell division. In the one-dimensional case, such changes can be conveniently expressed using the notion of L-systems. This provides an explanation of why L-systems work so well in modeling applications.

Molecular processes and pattern formation. We have illustrated modeling with cell complexes using examples of pattern formation in growing sequences of cells. Maxima of the concentration of a morphogen arise from an interplay between its local production and passive, diffusive transport, as in the model of Anabaena, or by reshuffling an existing or diffusely produced substance through active transport, as in the model of leaf margin. One question is whether these molecularly different mechanisms represent fundamentally different paradigms of pattern formation, or 
different implementations of a common principle. From the biological perspective it would also be interesting to know why such different mechanisms have evolved to create similar patterns.

Open problems. The confluence of L-systems and cell complexes provides a convenient framework for modeling one-dimensional developing structures. Modeling of higher-dimensional structures with dynamic cell complexes is substantially more difficult, and is a subject of ongoing research [5]. Difficulties extend to the visualization of three-dimensional models, where representations of the model's surface only provide partial information about the entire structure, and volumetric representations are often visually confusing.

Another problem open for further research concerns numerical methods for modeling structures with dynamic topology. Traditional formalisms for specifying and solving large systems of equations are based on matrix notation, which is not well suited for modeling multicellular organisms. First, matrices have fixed dimensions, so each time a cell divides, matrices describing the system globally have to be redefined. Second, the matrices are very sparse, since each cell can only be affected by a small number of neighboring cells due to the locality of interactions. Generalpurpose algorithms for solving systems of sparse equations use automatic techniques to identify which variables are connected through equations. However, constructing a sparse matrix and then identifying these connections represents unnecessary work, because a precise description of the connections between variables is already present in the topology of the complex. Thus, instead of expressing a structure using a matrix, and applying general methods for dealing with sparse matrices, it is better to operate directly on cell complexes $[4,29,30]$. The appropriate numerical methods have been devised in some contexts [12,31-33], but a more complete toolbox of numerical methods designed for dynamic cell complexes is needed.

Acknowledgments This note is an edited transcript of a presentation by PP at the workshop Pattern Formation in Morphogenesis (IHES, Bures-sur-Yvette, January 11-14, 2010). The authors thank Linda Pritchard for preparing the original transcript, and Lynn Mercer for comments on the manuscript. Insightful discussions and continuing collaboration with Eric Mjolsness on the mathematical foundations of biological modeling, Carla Davidson on the development of Anabaena, and Miltos Tsiantis and Adam Runions on the modeling of leaves, are gratefully acknowledged. The reported research has been supported by the Natural Sciences and Engineering Research Council of Canada and the Human Frontier Science Program.

\section{References}

1. A. Lindenmayer. Mathematical models for cellular interaction in development, Parts I and II. Journal of Theoretical Biology, 18:280-315, 1968.

2. L. De Floriani and A. Hui. Shape representation based on simplicial and cell complexes. In D. Schmalsteig and J. Bittner, editors, Eurographics 2007 State of the Art Reports, pages 63-87. The Eurographics Association, Prague, 2007.

3. L. Edelstein-Keshet. Mathematical Models in Biology. Random House, New York, 1988.

4. J. Chard and V. Shapiro. A multivector data structure for differential forms and equations. Mathematics and Computers in Simulation, 54:33-64, 2000. 
5. B. Lane, C. J. Harrison, and P. Prusinkiewicz. Modeling the development of multicellular structures using 3D cell complexes. In T. DeJong and D. Da Silva, editors, Proceedings of the 6th International Workshop on Functional-Structural Plant Models. University of California, Davis, 2010.

6. P. Prusinkiewicz. Developmental computing. In C. Calude, J. F. Costa, N. Dershiwitz, E. Freire, and G. Rozenberg, editors, Unconventional Computation. 8th International Conference, UC 2009, Lecture Notes in Computer Science 5715, pages 16-23. Springer, Berlin, 2009.

7. H. Weyl. Philosophy of Mathematics and Natural Science. Princeton University Press, Princeton, 1949.

8. P. Prusinkiewicz. Graphical applications of L-systems. In Proceedings of Graphics Interface '86 Vision Interface '86, pages 247-253, 1986.

9. R. Baker and G. T. Herman. Simulation of organisms using a developmental model, parts I and II. International Journal of Bio-Medical Computing, 3:201-215 and 251-267, 1972.

10. E. Coen, A.-G. Rolland-Lagan, M. Matthews, A. Bangham, and P. Prusinkiewicz. The genetics of geometry. Proceedings of the National Academy of Sciences, 101:4728-4735, 2004.

11. C. G. de Koster and A. Lindenmayer. Discrete and continuous models for heterocyst differentiation in growing filaments of blue-green bacteria. Acta Biotheoretica, 36:249-273, 1987.

12. P. Federl and P. Prusinkiewicz. Solving differential equations in developmental models of multicellular structures expressed using L-systems. In M. Bubak, editor, Computational Science - ICCS 2004 Part II, pages 65-72. Springer, Berlin, 2004. Lecture Notes in Computer Science 3037.

13. M. Hammel and P. Prusinkiewicz. Visualization of developmental processes by extrusion in space-time. In Proceedings of Graphics Interface '96, pages 246-258, 1996.

14. A. Lindenmayer. Adding continuous components to L-systems. In G. Rozenberg and A. Salomaa, editors, L Systems, Lecture Notes in Computer Science 15, pages 53-68. Springer-Verlag, Berlin, 1974.

15. P. Prusinkiewicz and A. Lindenmayer. The Algorithmic Beauty of Plants. Springer, New York, 1990. With J. S. Hanan, F. D. Fracchia, D. R. Fowler, M. J. M. de Boer, and L. Mercer.

16. R. Haselkorn. Heterocysts. Anual Review of Plant Physiology, 29:319-344, 1978.

17. R. Haselkorn. How cyanobacteria count to 10. Science, 282:891-892, 1998.

18. H.-S. Yoon and J. W. Golden. Heterocyst pattern formation controlled by a diffusible peptide. Science, 282:935-938, 1998.

19. O. Leyser. Auxin, self-organization, and the colonial nature of plants. Current Biology, 21:R331R337, 2011.

20. L. Gälweiler, C. Guan, A. Müller, E. Wisman, K. Mendgen, A. Yephremov, and K. Palme. Regulation of polar auxin transport by AtPIN1 in Arabidopsis vascular tissue. Science, 282:2226-2230, 1998.

21. A. Hay, M. Barkoulas, and M. Tsiantis. ASYMMETRIC LEAVES1 and auxin activities converge to repress BREVIPEDICELLUS expression and promote leaf development in Arabidopsis. Development, 133:3955-3961, 2006

22. E. Scarpella, D. Marcos, J. Friml, and T. Berleth. Control of leaf vascular patterning by polar auxin transport. Genes and Development, 20:1015-1027, 2006.

23. G. D. Bilsborough, A. Runions, M. Barkoulas, H. W. Jenkins, A. Hasson, C. Galinha, P. Laufs, A. Hay, P. Prusinkiewicz, and M. Tsiantis. Model for the regulation of Arabidopsis thaliana leaf margin development. Proceedings of the National Academy of Sciences, 108:3424-3429, 2011.

24. H. Jönsson, M. G. Heisler, B. E. Shapiro, E. M. Meyerowitz, and E. Mjolsness. An auxin-driven polarized transport model for phyllotaxis. Proceedings of the National Academy of Sciences, 103:1633-1638, 2006

25. R. S. Smith, S. Guyomarc'h, T. Mandel, D. Reinhardt, C. Kuhlemeier, and P. Prusinkiewicz. A plausible model of phyllotaxis. Proceedings of the National Academy of Sciences, 103:1301-1306, 2006. 
26. D. Draelants, J. Broeckhove, G. T. S. Beemster, and W. Vanroose. Pattern formation in a cell based auxin transport model with numerical bifurcation analysis. Preprint available at arXiv:1202.5161v1 [math.DS].

27. P. Sahlin, B. Söderberg, and H. Jönsson. Regulated transport as a mechanism for pattern generation: Capabilities for phyllotaxis and beyond. Journal of Theoretical Biology, 258:60-70, 2009.

28. J. Sethian. Level Set Methods and Fast Marching Methods: Evolving Interfaces in Computational Geometry, Fluid Mechanics, Computer Vision, and Materials Science. Cambridge University Press, Cambridge, 1999.

29. M. Desbrun, E. Kanso, and Y. Tong. Discrete differential forms for computational modeling. In E. Grinspun, P. Schröder, and M. Desbrun, editors, ACM SIGGRAPH 2006 Course Notes on Discrete Differential Geometry. ACM, New York, 2006.

30. R. Palmer and V. Shapiro. Chain models of physical behavior for engineering analysis and design. Research in Engineering Design, 5:161-184, 1993.

31. S. Parter. The use of linear graphs in Gauss elimination. SIAM Review, 3:119-130, 1961.

32. P. Prusinkiewicz, M. Allen, A. Escobar-Gutierrez, and T. DeJong. Numerical methods for transportresistance source-sink allocation models. In J. Vos, editor, Functional-Structural Modeling in Crop Production, pages 123-137. Springer, Dordrecht, 2007.

33. E. Sowell and P. Haves. Numerical performance of the SPARK graph-theoretic simulation program. In Proceedings of IBPSA Building Simulation '99, 1999. 